\title{
Some variant forms of the Poggendorff illusion and their implications for an explanation
}

\author{
R. H. DAY and E. J. STECHER \\ Monash University, Clayton, Victoria, Australia
}

\begin{abstract}
When with six widely different figures subjects adjusted one $45^{\circ}$ oblique bar or edge so that it appeared to be collinear with another, large misalignment errors of about the same size occurred in all figures, including one figure that consisted of two bars only (Experiment 1 ). When the ends of these bars were angled at $45^{\circ}$ to their axes and vertical in the figure, the errors were consistently greater than when the ends were square and oblique in the figure (Experiment 2). As well as calling into question some recent explanations of Poggendorff-type misalignment effects, these results suggest an alternative, more general explanation in terms of physical misalignment relative to a figure axis. This axis can be defined by elongated bars or lines, the ends of oblique bars, the direction of movement of the adjustable bar, or a combination of these.
\end{abstract}

The two experiments reported here followed some earlier observations made with figures similar to but not identical with those in Figure 1. The observations suggested that apparent misalignments between oblique bars occur strongly when a vertical rectangle and the bars are continuous with the ground rather than forming a figure (Figure 1C), when they form the arms of right angles with apexes either outward (Figure 1D) or inward (Figure 1E), and when the oblique features are the upper edges of vertical parallel bars (Figure 1F).

If confirmed, these observations not only would be of interest in their own right in adding to the body of misalignment effects to be explained, but would pose difficulties for some recent explanations of the effect. For example, the occurrence of a strong illusion with right-angle figures with apexes inward (Figure 1D) would be an embarrassment to Greist-Bousquet and Schiffman's (1981, $1985)$ proposal that the illusion is due to a Müller-Lyerinduced contraction between the oblique elements. In Figure 1D, apparent expansion between the angles along the axis of the bisectors would be expected (see below). This difficulty would be compounded if, as the preliminary observations indicated, apparent misalignment in Figure 1D proved to be as great as that in Figure 1E, in which apparent contraction would be expected. Apparent misalignment in either figure would also pose a problem for Day's (1989) argument that apparent misalignments occur when there is a physical misalignment of the oblique relative to a clearly delineated main axis of the figure like that in Figures 1A, 1C, and 1F. There

This research was supported by a grant to $\mathbf{R}$. H. Day from the Australian Research Council. The authors gratefully acknowledge the invaluable assistance given by Mike Durham, Rosemary Williams, and Vladimir Kohout in computer programming, illustration, and photography, respectively. Requests for reprints should be sent to $\mathbf{R}$. H. Day, Department of Psychology, Monash University, Clayton, Victoria 3168 , Australia.
A

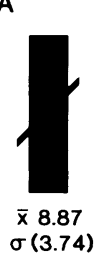

B

C

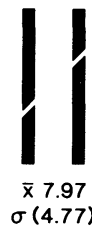

E

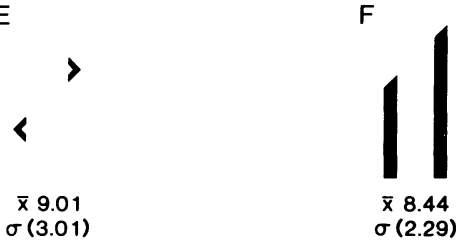

Figure 1. The stimulus figures for Experiment 1. The mean apparent misalignments of the oblique elements (bars or edges) are also shown with their standard deviations.

are no such delineated axes in Figures $1 \mathrm{D}$ and 1E. However, as will be noted below, the axis of a figure can be indicated by the ends of the bars and the direction of their movement during adjustment to alignment, as well as by parallel lines or edges as in Figures 1A, 1C, and 1F.

The purpose of the first experiment was to confirm (or disconfirm) the earlier observations using Figures 1C-1F and to compare them with those for the more conventional form of the Poggendorff figure in Figure 1A and its 
A

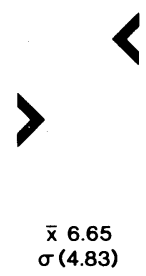

D

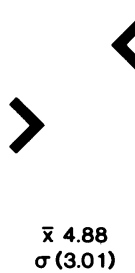

B

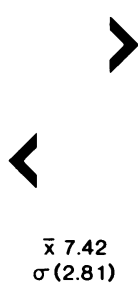

$E$

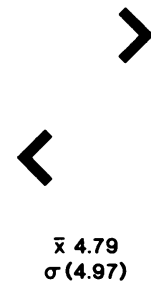

C

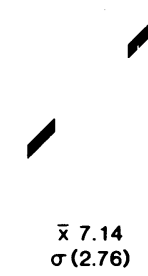

$\mathbf{F}$

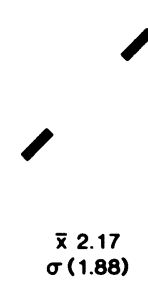

Figure 2. The stimulus figures for Experiment 2. The mean apparent misalignments of the oblique bars are also shown with their standard deviations.

"parallel-less" version in Figure 1B. For the earlier observations, the ends of some bars were at right angles to their long axes and oblique in the figure (squared ends) and the ends of the others were at $45^{\circ}$ to the long axes and vertical in the figure (angled ends). This inconsistency was removed in Experiment 1 by making all bar ends angled as in Figure 1. However, the impression was gained that the angled ends gave rise to greater effects than did the squared ends in the earlier observations. This impression was tested in Experiment 2, in which the bar ends were either angled or squared as shown in Figure 2. As will be noted below, the impression of a greater effect with angled ends was confirmed and has proved to be a key in formulating an explanation of misalignment effects.

\section{EXPERIMENT 1}

\section{Method}

Subjects. There were 12 subjects, 8 women and 4 men, recruited from among undergraduate and graduate students.

Apparatus. The stimulus figures were presented on the screen of a personal computer (Terran T20) fitted with a video-graphics adaptor. The screen measured $27.5 \mathrm{~cm}$ horizontally and $20.4 \mathrm{~cm}$ vertically. Subjects were seated at a table without head restraint and instructed to keep their heads upright throughout. The viewing distance was about $114 \mathrm{~cm}$, so $1 \mathrm{~cm}$ subtended a visual angle of about $0.50^{\circ}$. The computer was programmed to generate the stimulus figures, present them in a different random order for each subject, vary the starting position of the right oblique bar, and record, average, and store individual scores. The right oblique element could be moved vertically in either direction by pressing one of two marked keys on a keyboard. After completion of each adjustment to apparent alignment, the subject pressed a third key to bring up the next figure in the sequence.

Stimulus figures. The six stimulus figures are shown in Figure 1. The linear and angular dimensions of all six were derived from the more conventional form of the Poggendorff figure in Figure 1A. In this figure, the rectangle was $124 \mathrm{~mm}$ high and $30 \mathrm{~mm}$ wide, and the $45^{\circ}$ oblique bars were $14 \mathrm{~mm}$ long and $4 \mathrm{~mm}$ thick. The oblique distance between the bars when they were aligned was $42 \mathrm{~mm}$. In Figures $1 \mathrm{C}$ and 1F, the enclosed rectangular space was the same as the black vertical rectangle in Figure 1A, and the vertical parallel bars enclosing it were $10 \mathrm{~mm}$ wide. In Figure 1F, the right and left bars were 124 and $72 \mathrm{~mm}$, respectively, along their outer vertical edges, and their $45^{\circ}$ upper edges were the same in length and separation as were the oblique bars in the other figures. When the right oblique upper edge of this figure was adjusted up or down, the whole bar was lengthened or shortened from its fixed base. In Figures 1D and 1E, the oblique bars formed a right angle with the other bars. The ends of all bars were angled but vertical in the figure (see above).

Procedure. Throughout, the task of the subject was to adjust the oblique bar or edge on the right of each figure so that it appeared to be perfectly aligned with that on the left. Four adjustments were made for each figure, starting at $\mathbf{1 8 . 7} \mathrm{mm}$ above the position of exact alignment and $18.7 \mathrm{~mm}$ below that position in alternation. The order in which the figures were presented was randomized differently for each subject, and all four adjustments were completed with one figure before the next was presented. The score for each figure was the mean vertical difference in millimeters between the positions of apparent and exact alignment based on the four adjustments. Scores in the direction to compensate for the Poggendorff misalignment effect were treated as positive and those in the opposite direction as negative.

\section{Results and Discussion}

The mean misalignment scores and their standard deviations are shown in Figure 1. They ranged from $9.01 \mathrm{~mm}$ for Figure 1E to $6.47 \mathrm{~mm}$ for Figure 1D. Separate $t$ tests showed that all means were significantly different from zero at $p<.001$. A one-way analysis of variance (ANOVA) showed that the means were not significantly different from each other $[F(1,11)=.90, p<.40]$. Thus the misalignment illusion was as great as that in the more conventional figure when the vertical rectangle and oblique bars were continuous with the background, when the figure consisted of only two oblique bars or of two right angles with apexes outward or inward, and when it consisted of two vertical bars with oblique ends.

For the reasons pointed out above, the absence of any difference between the means for the two right-angle figures is damaging for the explanations of the illusion in terms of reduced apparent extent between the obliques (Greist-Bousquet \& Schiffman, 1981, 1985) and in terms of their physical misalignment relative to the delineated axis of the figure (Day, 1989). The equally strong effect with only two oblique bars is likewise damaging for this explanation. It can be noted that, relative to the figure with parallels, both Day (1973) and Goldstein and Weintraub (1972) found only a very small (but significant) effect for a figure consisting of two $45^{\circ}$ oblique lines. The results of the second experiment bear closely on the question of why the effect was much greater with a figure consisting of two oblique bars with vertical ends as in Figure 1B than it was with two lines.

\section{EXPERIMENT 2}

For the reasons stated in the introduction, the purpose of Experiment 2 was to confirm or check whether the form of the ends of the bars, angled or squared, influenced the size of the misalignment effect. Three figures used 
in Experiment 1-Figures 1B, 1D, and 1E-with angled ends and three with squared ends were presented.

\section{Method}

The source of the 12 new subjects ( 8 men and 4 women), the apparatus and viewing conditions, and the procedure were the same as before. The six stimulus figures are shown in Figure 2. Figures 2A, 2B, and $2 \mathrm{C}$, with angled ends, were the same as those in Experiment 1, and Figures 2D, 2E, and 2F were the same except for the square ends of the bars.

\section{Results and Discussion}

The mean misalignment scores for the six figures are shown with their standard deviations in Figure 2. The mean for each angled-end figure was greater than its squared-end partner. In the case of the two figures consisting of only two bars, the mean for the angled-end bars was about the same as those for the two similar right-angle figures, whereas that for the squared-end bar was much less than that for the two similar right-angle figures. Separate $t$ tests showed that all means were significantly greater than zero-those for Figures 2A, 2B, 2C, and 2E at $p<.001$, and those for Figures 2D and 2F at $p<.01$. A $2 \times 3$ ANOVA in which the main factors were the bar ends (angled or squared) and figure (two bars, right angles with apexes outward or right angles with apexes inward) showed that whereas the first of these was significant $[F(1,11)=30.92, p<.01]$, the second was not $[F(1,11)=1.35, p>.05]$. However, the interaction between the two factors was also significant $[F(2,22)=$ 5.43, $p<.05]$. A Newman-Keuls comparison of the means showed that this was due to the smaller misalignment effect with Figure 2D as opposed to Figures 2E and $2 \mathrm{~F}$, for the squared-end figures.

It can be concluded that apparent misalignment with oblique bars is greater when the ends of the bars are angled and therefore vertical with respect to the figure itself than it is when they are squared and oblique in the figure. With the latter, the effect proved to be consistently less than it did with the former.

\section{GENERAL DISCUSSION}

The occurrence of apparent misalignment effects with collinear features continuous with the background as in Figure $1 \mathrm{C}$ or in the form of edges as in Figure 1F is not particularly surprising. Nevertheless, these effects are of interest in that they demonstrate the generalization of the Poggendorff illusion to features other than collinear lines. What is surprising are the strong effects in Figures 1B, 1D, and 1E, in which the aligned features were bars rather than the more usual lines. In Figure 1B, which consisted simply of two bars, the illusion was of about the same size as that in the more conventional Figure 1A. In the case of two thin lines rather than bars, the illusion is very slight and much less than that in the conventional figure (Day, 1973; Goldstein \& Weintraub, 1972). In Figures 1D and 1E, the effects, as strong in statistical terms as those in the more conventional Figure 1A, are contrary to ex- pectations from the explanations proposed by Greist-Bousquet and Schiffman $(1981,1985)$ and Day (1989), as already pointed out. Finally, and equally surprising, when in the second experiment the ends of the aligned bars were angled but vertical in the figure, the illusions of misalignment were consistently greater than when the ends were squared and oblique in the figure. As far as is known, this has not been reported before.

The apparent misalignment for the six figures in Figure 1 proved not to be significantly different from each other. The question can therefore be asked whether there is any feature common to these quite diverse forms that might suggest a more general explanation capable of encompassing the now wide range of misalignment illusions. We believe that there is, and tentatively propose the following.

In all figures in Figure 1, the aligned feature (bars or edges) are displaced both vertically and laterally in the figure. However, the vertical dimension is rendered more salient in three ways: it is clearly delineated by vertical bars, as in Figure 1A, 1C, and 1F; by the vertical ends of the bars, as in Figure 1B, 1D, and 1E; and by vertical movement of one bar during adjustments to alignment. We suggest that alignment of one bar with the other in their oblique axis is compromised in all figures by the more elevated position of the bar or edge on the right relative to the vertical axis when the latter is actually delineated by vertical features such as lines or bars, by the ends of oblique bars, or by the vertical movement of the adjustable bar. When one or more of these verticaldelineating conditions prevail, the uppermost bar appears slightly too high in the vertical axis for apparent alignment. In short, when an axis of a figure is clearly defined by bars or lines, by the ends of the bars, or by direction of movement of the adjustable feature, the displacement of the bars relative to that axis will compromise apparent misalignment in another axis. We suggest also that if one of these axis indicators is in conflict with another, the illusion will be reduced. Thus we suggest that in Figures 2C, 2D, and 2E the squared ends were at variance with the vertical movement, thereby reducing the size of the effect relative to that in Figures 2D, 2E, and $2 F$.

Of course, this compromise would be expected also to obtain when the aligned features are displaced relative to any axis clearly defined by bars, lines, ends of features, or direction of adjusting movements.

Space does not permit a more detailed statement of this explanation of the Poggendorff misalignment effect and its variants. It is sufficient to note that on first consideration it appears to have wide application and lends itself to systematic testing. However, it can be noted that it is a modification and refinement of the explanation proposed earlier (Day, 1989).

\section{REFERENCES}

DAY, R. H. (1973). The oblique line illusion: The Poggendorff effect without parallels. Quarterly Journal of Experimental Psychology, 25, 535-541.

DAY, R. H. (1989). Natural and artificial cues, perceptual compromise and the basis of veridical and illusory perception. In D. Vickers \& P. L. Smith (Eds.), Human information processing: Measures, mechanisms and models (pp. 107-129). Amsterdam: North-Holland.

Goldstein, M. B., \& Weintraub, D. J. (1972). The parallel-less Poggendorff: Virtual contours put the illusion down but not out. Perception \& Psychophysics, 11, 353-355.

Greist-Bousquet, S., \& Schiffman, H. R. (1981). The Poggendorff illusion: An illusion of linear extent? Perception, 10, 155-164.

Greist-Bousquet, S., \& Schiffman, H. R. (1985). Poggendorff and Müller-Lyer illusions: Common effects. Perception, 14, 427-447.

(Manuscript received June 22, 1991.) 